\title{
Dynamic Resource Allocation with a Soft Handover Procedure for Application in a Broadband System
}

\author{
Gabriele Lupo, Roberto Cautelier, Maria-Gabriella Di Benedetto, Francesco Malena \\ Università degli Studi di Roma La Sapienza - Dipartimento INFOCOM \\ Via Eudossiana, 18,00184 Roma - Italy \\ e-mail: bob@wsaquila.ing.uniromal.it
}

\begin{abstract}
This work investigates the advantages of adopting a Soft HandOver (SHO) procedure with a TDD-TDMA-OFDM access method in a WLAN system, when broadband mobile users are considered. Reference is made to the MEDIANACTS system scenario extended to a multi-cell environment.

A particular distributed queue packet reservation multiple access scheme with priorities, called PAMA (Priority Assignment Multiple Access) is considered. Simulation results carried out in the present investigation indicate that SHO outperforms Hard HO (HHO), in terms of packet loss and time delay, and its performance approaches that of the fixed users case. In fact, SHO exploits the overlapping area between adjacent cells permitting users to be simultaneously served by two Base Stations (BSs) instead of a single one. As a consequence, the overall offered traffic is better distributed among the BSs of the system, resulting into a more efficient resource allocation.
\end{abstract}

\section{Introduction}

The system analyzed in this work is an extension of the MEDIAN-ACTS system to a multi-cell environment. The MEDIAN system is designed for multimedia communications in a Wireless Local Area Network (WLAN), in which broadband users transmit ATM-like packets using a TDD-TDMA-OFDM (Time Division DuplexTime Division Multiple Access-Orthogonal Frequency Division Multiplexing) technique [1]. High traffic is offered to each Base Station (BS) by a small number of multimedia users.

An extension of the MEDIAN architecture to include mobility with a particular Hard HandOver (HHO) procedure was proposed in [2]. The effects of adopting a Soft HandOver (SHO) procedure in such a system are analyzed in this paper.
A single cluster is considered in this work. Each Base Station (BS) in the cluster is supposed to use a different frequency band, so that there is no frequency reuse between adjacent cells. It is also assumed that packet exchanging between BSs is instantaneous, and that packet routing in a cluster is centrally controlled by a higher layer entity called Mobility Manager (MM). Each BS works as a buffered switch connected through an optical fiber cable to the other buffered switches (the other BSs) belonging to the same cluster and to the MM.

In accordance to the MEDIAN MAC (Medium Access Control), a TDD-TDMA-OFDM technique is adopted. A particular resource allocation protocol, referred as PAMA (Priority Assignment Multiple Access), is considered; PAMA allows a different priority to be dynamically obtained by the users according to the evolution of their packet generation. The great advantage of this scheme, which makes it particularly appropriated for broadband applications, is that slot allocations are very flexible, depending on the offered traffic.

The proposed SHO procedure exploits the overlapping area between adjacent cells, permitting users to be simultaneously served by two BSs instead of a single one. A user served by two BSs transmits different packets on the two available links, according to the centralized controlled slot allocations. In this way, the traffic offered by a Mobile Station (MS) located in the SHO region is split between the two BSs. Therefore, the overall offered traffic is better distributed among the BSs of the system, resulting into a more efficient resource allocation in terms of packet loss and time delay.

This work is organized as follows. In Section 2, the PAMA resource allocation protocol will be described. Section 3 will be focused on the proposed SHO scheme. In Section 4, simulation results obtained for the case of two cells with moving video users will be analyzed. Finally, Section 5 will be dedicated to the conclusions. 


\section{Priority Assignment Multiple Access (PAMA)}

In agreement with MEDIAN, in the proposed system a frame is divided into an uplink and a downlink channel, following a TDD-TDMA technique adopting the OFDM modulation. In each frame, the first 32 time slots are dedicated to the downlink channel and second 32 time to the uplink channel, as shown in Fig.1. The last downlink slot, called Broadcast slot, is used by the BS to transmit the Broadcast Cell which defines resource allocation in the subsequent frame. The first uplink time slot is called Signalling slot and is used by MSs to forward a resource access request.

The Signalling slot is shared among different active users supposing that each BS assigns a different OFDM subcarrier to each user served by it. Each active user is allowed to send an ON/OFF flag per Signalling slot, to inform the BS about a resource allocation request (flag sets to $\mathrm{ON}$ ). Since only a small fraction of the whole set of subcarriers is needed, an ON flag can be simply detected by measuring energy on the corresponding subcarrier. The user sets the Signalling flag to ON every time it needs to send at least one packet. Obviously, one flag per frame is not enough to inform the BS about the number of slots needed by a user. Therefore, an additional control information, called Queue Signalling message (QS), is introduced. The QS can assume the values $+1,0,-1$ to represent the need of an increase, an invariance or ether a decrease of the number of slots to be allocated, respectively. The possibility of sending one packet allows the user to send the QS formed by the value of two bits attached to the packet, using a classic piggy-back mechanism. The QS messages allow the BS to estimate the size of the transmission buffer of each user, called the Estimated Priority of that user (EP( $i)$ for the $i$-th user). The number of slots assigned by the BS to the $i$-th user is calculated as the number of slots required by the user, $\operatorname{EP}(i)$. If the total number of slots to be assigned is greater than the number of available slots, the actual number of allocated slots is obtained by normalization. In any case at least one slot is allocated to users with $\operatorname{EP}(i)>0$.

The allocation algorithm tends to assign a number of slots which follows as closely as possible the number of required slots.

\section{Soft Handover Scheme}

SHO is carried out by a Mobile Controlled and Forward $\mathrm{HO}$ procedure. The temporization of the
$\mathrm{HO}$ procedure is illustrated in Fig. 2 for the case of a user moving from the OldBS to the NewBS. The figure shows the qualitative evolution of the received signal levels relative to the 'handovering' MS. Figure 3 illustrates the corresponding MS position in the cluster. In what follow the temporization is explained:

- $\mathbf{t}_{0}$ : the MS is situated inside the base-cell (hexagonal) of the OldBS and is served only by it. The base-cell of a BS is the region defined by the points in which the level of the signal received from it is greater than those of the signals coming from the neighbor BSs.

- $\mathbf{t}_{\mathbf{1}}$ : the MS enters the base-cell of the NewBS; it forwards the $\mathrm{HO}$ request to the NewBS without releasing the old link. While in the $\mathrm{SHO}$ area (blacked), the MS transmits the Signalling flag to both BSs using the corresponding signaling slots, maintaining two simultaneous links. The two links are used by the MS to transmit different packets. After receiving the $\mathrm{HO}$ request, the NewBS can obtain the in-coming user profile from the OldBS. In addition, the Home Location Register of the MS is up-dated, and the NewBS assigns the MS a new subcarrier for resource allocation requests, which is communicated by means of a specific Broadcast Cell. Note that the SHO area does not coincide with the overlapping cell area.

- $\mathbf{t}_{2}$ : the MS crosses the extended-cell frontier of the OldBS (circular line in Fig.3) and consequently releases the old link. The extended-cell of a BS is the region defined by the points in which the level of the signal received from it is greater than a given threshold value ( $\mathrm{L}_{2}$ in Fig. 2 ).

Finally, after $t_{2}$ The MS is situated in the basecell of the NewBS and continues the transmission only with this BS.

It has been assumed that the sojourn times of a generic MS in a base-cell and in a SHO area are exponentially distributed, with respective average values named THO (Time of HO) and TSHO (Time of SHO). The THO corresponds to the average time interval between successive $\mathrm{HO}$ requests of a given MS. The TSHO is equal to the average time during which a user maintains two simultaneous links.

These two values characterize the user mobility. The ratio TSHO/THO is a system parameter since it depends on the ratio between the SHO area and base-cell area. 


\section{Simulation Results}

The simulated system is composed of two cells with moving video users, modeled according to the H.263 standard [3], transmitting with average bit rate of 3 Mbps. Each user is supposed to communicate only with another user situated in the system and to be permanently active.

Channel characteristics are described in Tab.I. Only the uplink channel is considered and performances are evaluated in terms of packet dropping and packet delay.

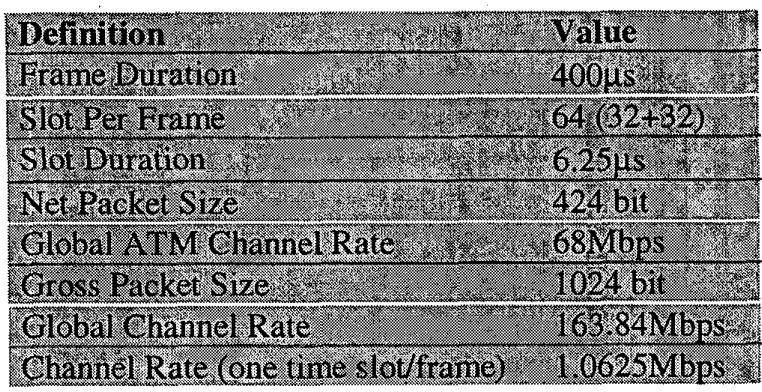

Table I Channel parameters

The ratio between THO and TSHO is fixed. Consequently, user mobility can be completely characterized by a single parameter named HOP, defined by the following relation:

$\mathrm{THO}+\mathrm{TSHO}=1 / \mathrm{HOP}$

Figure 4 shows the obtained average packet dropping percentage as a function of the average number of users in a cell, in the cases of no mobility, HHO procedure and SHO. Results are obtained with HOP $=1.5 \cdot 10^{-4}$ and $\mathrm{TSHO} / \mathrm{THO}=1 / 4$. The SHO allows having 9 video users per BS with an average packet dropping below about $1 \%$. Compared to HHO the improvement is in the order of about $2.5 \%$. The performance improvement is also clear in terms of time delay, as can be observed in Fig.5. There, only the time delay of the successfully transmitted packet has been taken into account, and therefore even greater differences will be expected by also considering the lost packets.

Figure 6 illustrates the behavior of the average packet dropping as the degree of user mobility varies, for the case of 8 and 9 users per cell. The degree of user mobility is expressed by the HOP parameter. It can be observed that the packet dropping diminishes, as the user speed increases. This behavior is a consequence of a better distribution of the traffic between the BSs. This effect was deeply analyzed in a previous paper [4], where a theoretical model was proposed to explain the increase of resource allocation efficiency as the user speed increases.

Figure 7 shows the average packet dropping as a function of the cell overlapping area (expressed by the ratio TSHO/THO) for different values of the number of users per BS. As expected, greater ratios correspond to better performance. Moreover, even for quite low values such as TSHO/THO $=1 / 10$ the packet dropping is much lower than what obtained with HHO (about $1.2 \%$ in the case of 9 users per cell).

\section{Conclusions}

In this work, the advantages of adopting a Soft HandOver (SHO) procedure with a TDD-TDMAOFDM access method in a WLAN system with broadband mobile users have been investigated.

Simulation results have been presented which indicate that SHO outperforms Hard HO in terms of packet loss and time delay, and its performance approaches that of the fixed users. The proposed SHO exploited the overlapping area between adjacent cells permitting a better distribution of the overall offered traffic among the BSs of the system, and resulted into a more efficient resource allocation.

\section{References}

[1] European Community ACTS MEDIAN project AC006, "Wireless Broadband CPN/LAN for Professional and Residential Multimedia Applications," Final System Design, Deliverable MD3, CEC reference \#AC006/MED/PAR/DS/R/054/b1, December 1996.

[2] F. Malena, M. Del Buono, M.G. Di Benedetto, "Dynamic resource allocation with a novel handover procedure for application in a broadband system", in Proc. of $3^{\text {rd }}$ ACTS Mobile Communication Summit, June 1998, Rhodes, Greece.

[3] M. Del Buono, L. Hanzo, P. Cherriman, "Oscillation-scaled histogram-based Markov modelling of video codecs", in Proc. of PIMRC'97, Sept. 1997, Helsinki, Finland.

[4] G. Lupo, N. Blefari-Melazzi, R. Cautelier, M.G. Di Benedetto, "A model for cellular systems with broadband users: effet of users speed on resource allocation efficiency", Proc. of $4^{\text {rh }}$ ACTS Mobile Communication Summit, June 1999, Sorrento, Italy. 


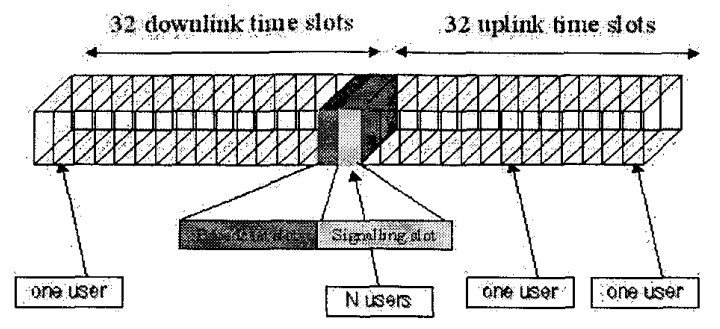

Figure 1 Frame structure in the PAMA scheme.

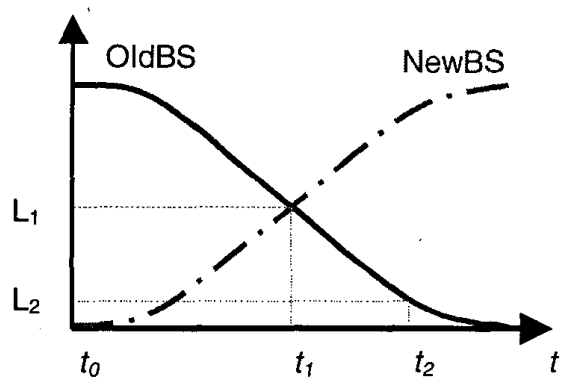

Figure 2 Signal levels at the MS moving from OldBS to NewBS.

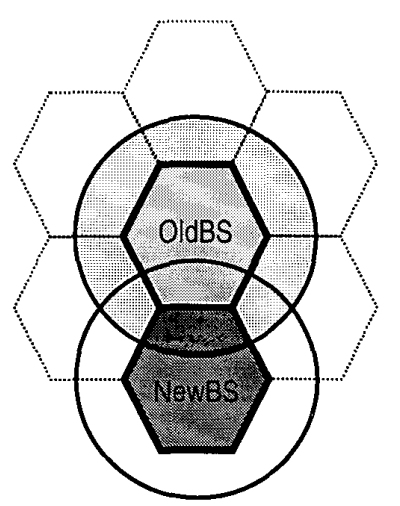

Figure 3 Base-cell and extended-cell.

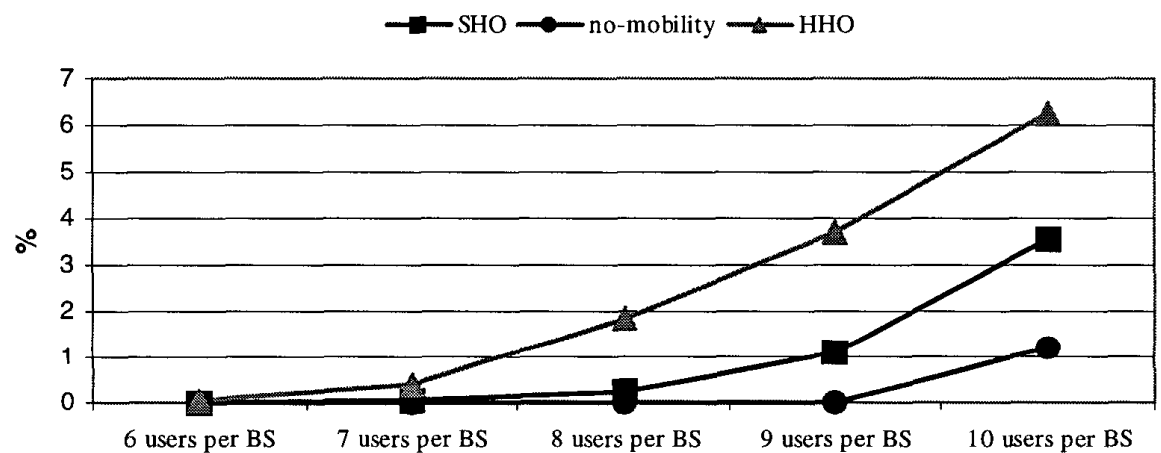

Figure 4 Average uplink packet dropping percentage vs. number of users in the system.

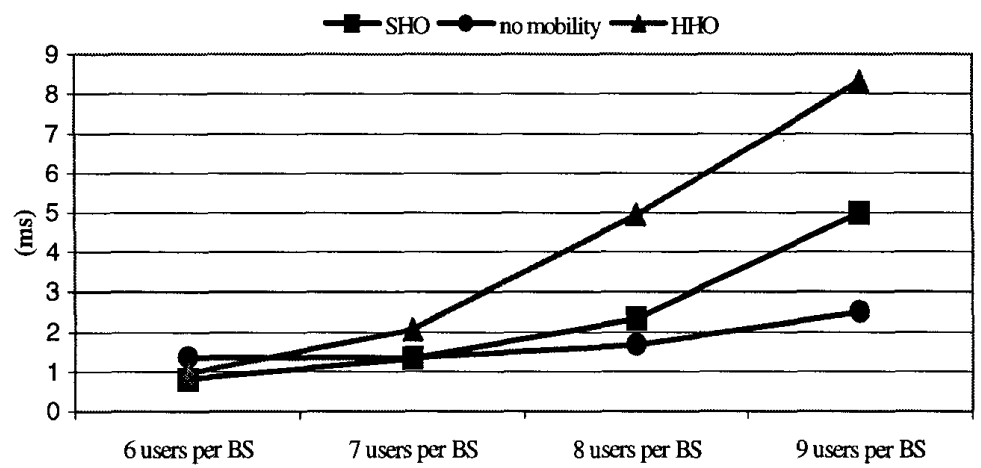

Figure 5 Average uplink packet delay vs. number of users in the system. 


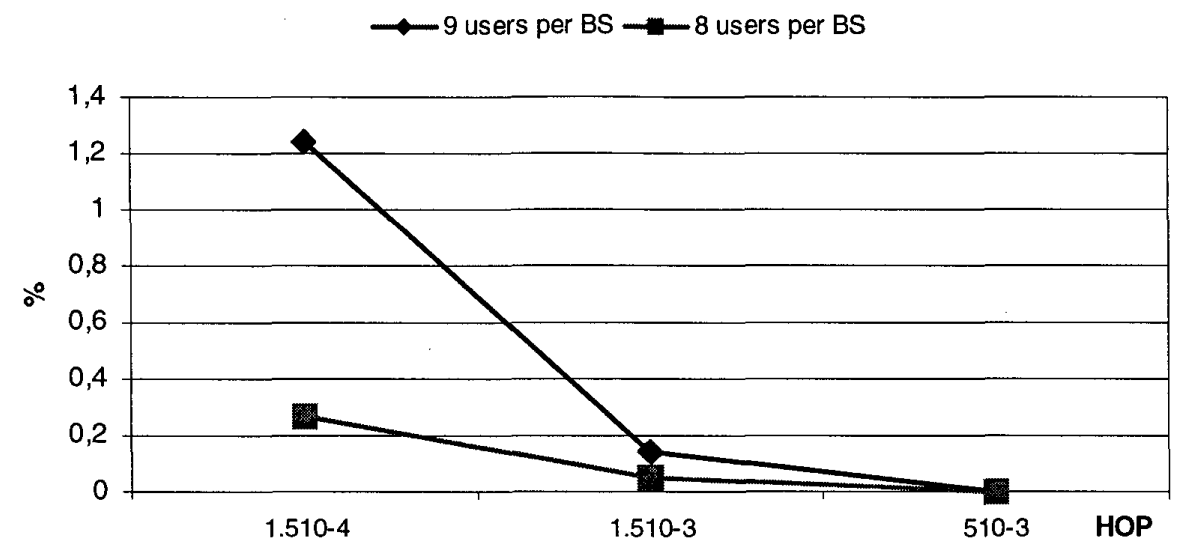

Figure 6 Average uplink packet dropping varying the degree of user mobility for 8 and 9 users per cell.

$\longrightarrow 7$ users per BS -8 users per BS -9 users per BS

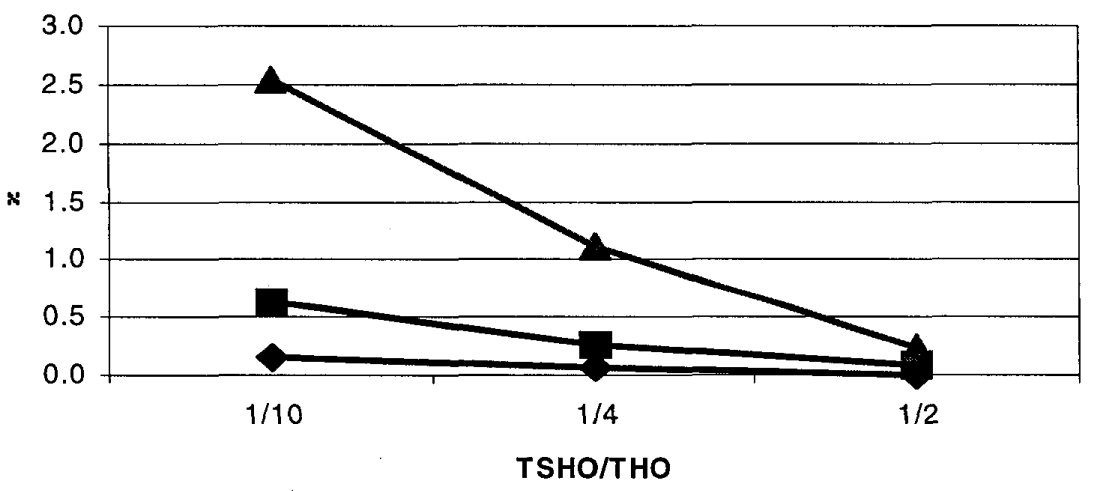

Figure 7 Average uplink packet dropping vs ratio between SHO area and base-cell area. 